\title{
Investigating the Distribution of Stable Paramagnetic Species in an Apple Seed Using X-Band EPR and EPR Imaging
}

\author{
Kouichi Nakagawa ${ }^{1 *}$ and Boris Epel ${ }^{2}$ \\ ${ }^{1}$ Division of Regional Innovation, Graduate School of Health Sciences, Hirosaki University, 66-1 Hon-Cho, Hirosaki 036-8564, JAPAN \\ ${ }^{2}$ Department of Radiation and Cellular Oncology, The University of Chicago, MC1105, 5841 S. Maryland Ave, Chicago, IL 60637-1463, USA
}

\begin{abstract}
This study investigated the location and distribution of paramagnetic species in apple seeds using electron paramagnetic resonance (EPR) and X-band (9 GHz) EPR imaging (EPRI). EPR primarily detected two paramagnetic species per measured seed. These two different radical species were assigned as stable radicals and $\mathrm{Mn}^{2+}$ species based on the $g$ values and hyperfine components. The signal from the stable radical was noted at $\boldsymbol{g} \approx \mathbf{2 . 0 0}$ and was strong and relatively stable. The subsequent noninvasive EPRI of the radical present in each seed revealed that the stable radicals were located primarily in the seed coat, with very few radicals observed in the cotyledon of the seed. These results indicate that the stable radical species were only found within the seed coat, and few radical species were found in other seed parts.
\end{abstract}

Key words: EPR, apple, seed, EPR imaging, free radical

\section{INTRODUCTION}

Electron paramagnetic resonance (EPR) spectroscopy is a very sensitive technique for the noninvasive detection of free radicals both in vitro ${ }^{1-3)}$ and in vivo ${ }^{4)}$. The EPR spectrum appears either as an asymmetric line-shape or as a series of multiple overlapped lines, depending on the sample being assessed ${ }^{1)}$. $250 \mathrm{MHz}$ EPR imaging (EPRI) has been developed to investigate the spatial distribution and relaxation of spin probes administered in small animals ${ }^{4)}$. Similar technological advances have also produced an $\mathrm{X}$-band (9 GHz) EPRI, which has a higher spatial resolution, greater sensitivity, and lower sample volume than low frequency EPRI. The application of noninvasive EPRI and EPR spectroscopy can provide detailed information regarding the location and concentration of paramagnetic species (e.g., transition metal ions, transition metal complexes, and stable organic radicals) in naturally occurring biological samples.

Apples are a popular and healthy fruit, and apple seeds are used in various applications. Apple seed oil is produced as a byproduct of juice processing, and is made from raw apple pomace. It is used as a fragrance, as well as for conditioning hair and calming skin inflammation. Studies examining the fatty acid composition, physicochemical properties, and biological attributes (antioxidant and in vitro cytotoxicity) of apple seed oil have produced interesting findings ${ }^{5)}$. For example, oleic and linoleic acids were found to be the dominant substances comprising fatty acids in apple seed oil $(46.50 \% \text { and } 43.81 \% \text {, respectively })^{5,6)}$. The physicochemical properties of apple seed oil were found to be comparable with those of edible food oil, indicating improved stability and the potential for broad application in both the food and pharmaceutical industries ${ }^{6)}$. The in vitro cytotoxic activity of apple seed oil against specific cell lines also suggested its potential as an anticancer agent. Little is known regarding the effects of the presence of endogenous paramagnetic species (e.g., $\mathrm{Mn}^{2+}$ ) and organic radicals in the seeds of apples. EPRI could be a useful tool for obtaining such information.

In the present study, paramagnetic species in chemically untreated apple seeds and seeds parts were examined using X-band EPR and noninvasive EPRI. EPR spectroscopy detected two types of paramagnetic species in the seeds, whereas the distribution of radicals within the seeds was observed using EPRI. The localization and concentration of stable radical species within the seeds are discussed.

\footnotetext{
* Correspondence to: Kouichi Nakagawa, Division of Regional Innovation, Graduate School of Health Sciences, Hirosaki University, 66-1 Hon-Cho, Hirosaki 036-8564, JAPAN

E-mail: nakagawa@ hirosaki-u.ac.jp

Accepted Ocotber 20, 2016 (received for review July 29, 2016)

Journal of Oleo Science ISSN 1345-8957 print / ISSN 1347-3352 online

http://www.jstage.jst.go.jp/browse/jos/ http://mc.manusriptcentral.com/jjocs
} 


\section{EXPERIMENTAL}

\subsection{Samples}

Fuji apples were purchased from a supermarket located in the far north (Hirosaki, Aomori Prefecture) of the main island of Japan in January 2016, and were used without additional treatment. The apples were harvested in the fall of 2015. For the measurements, seeds $(\sim 0.0316 \mathrm{~g} / \mathrm{seed})$ were sequentially inserted into an EPR tube (outer diameter 5.0 mm, inner diameter $4.0 \mathrm{~mm}$, Wilmad LabGlass, USA) .

A spin probe reagent, 4-hydroxy-2,2,6,6-tetramethylpiperidin-1-oxyl(TEMPOL), and 1,1-diphenyl-2-picrylhydra$\mathrm{zyl}(\mathrm{DPPH})$ radicals were purchased from Nacalai Tesque, Inc. (Kyoto, Japan) and were used as received. In these cases, the same DPPH sample was used as a reference sample.

For phantom measurements, the DPPH radicals were attached to a plastic plate $(\sim 3 \mathrm{~mm}$ wide) using commercially available, synthetic rubber-type glue. The phantom was composed of four DPPH point samples configured in the layout on the plate shown in Fig. 1, with the first and second pairs of DPPH positioned 2-4 mm apart. For each measurement, the DPPH phantom was inserted into an EPR tube or an EPR rod.

\subsection{EPR measurements}

A JEOL RE-3X 9 GHz EPR spectrometer (JEOL Ltd., Tokyo, Japan) was used for continuous wave (CW) measurements. The system was operated at $9.43 \mathrm{GHz}$ using a 100-kHz modulation frequency. All CW EPR spectra were

\section{DPPH phantom}

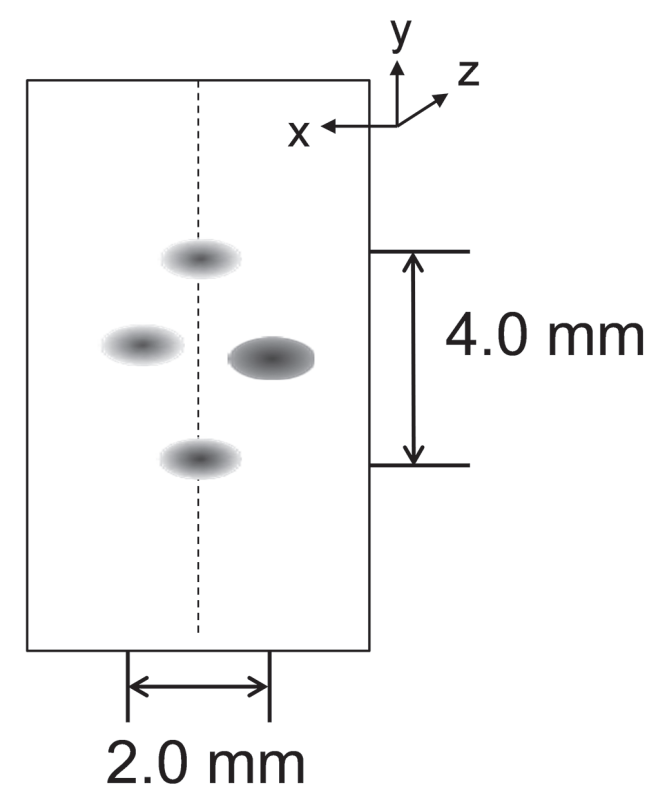

Fig. 1 Schematic illustration of the DPPH phantom. The coordinates of the magnetic field gradients are also indicated. obtained in a single scan. Typical CW EPR settings were as follows: microwave power, $5 \mathrm{~mW}$; time constant, $0.1 \mathrm{~s}$; sweep time, 4 min; magnetic field modulation, $0.32 \mathrm{mT}$; and magnetic field sweep width, $10 \mathrm{mT}$.

\subsection{EPR imaging measurements}

A commercially available JEOL RE-3X 9 GHz EPR spectrometer was modified for use as an EPR imager by adding magnetic-field gradient coils (Yonezawa Densen Ltd., Yonezawa, Japan) and their power supplies. For $9 \mathrm{GHz}$ EPR imaging, the $\mathrm{x}$ - and $\mathrm{y}$-axes of the gradient coils in antiHelmholtz coil configurations were used. To avoid overheating, the gradient coils were cooled with water $\left(16^{\circ} \mathrm{C}\right)$. Takasago BWS 60-5 bipolar power supplies (Takasago Ltd., Tokyo, Japan) were used. The maximum available field gradient along the $\mathrm{x}$ - and y-axes was $3.3 \mathrm{mT} / \mathrm{cm}$. The magnetic field of the EPR spectrometer was current stabilized to perform EPR imaging. The field-based control is incompatible with the application of the magnetic field gradients. All CW EPR spectra were obtained in a single scan. The instrument and the gradients were controlled using the SpecMan4EPR (FeMi Instruments LLC, USA) software. All measurements were performed at ambient temperature.

\subsection{EPR imaging and imaging data processing}

We used 16 equal angle spaced projections obtained with maximum gradient of $\sim 3.3 \mathrm{mT} / \mathrm{cm}$. The first-derivative EPR spectra were numerically integrated to obtain the corresponding absorption spectra. The two-dimensional (2D) images were reconstructed using the back-projection algorithm from the EPR-IT software package from the Center for EPR Imaging in vivo Physiology at the University of Chicago $^{7)}$. The data were processed in the MathWorks MATLAB $^{\mathrm{TM}}$ environment.

\section{RESULTS AND DISCUSSION}

\subsection{EPR and EPRI of DPPH phantom}

Phantom experiments were performed to examine the capability of the EPRI. Figure 2 shows the CW EPR spectrum of the DPPH phantom. The sweep width was $5 \mathrm{mT}$ with a peak-to-peak linewidth $\left(\Delta \mathrm{H}_{\mathrm{pp}}\right)$ of approximately 0.14 mT. A slightly broader single line of the EPR spectrum indicates that the quantity of DPPH applied on the plate was slight at off-line positions.

Figure 3 shows the reconstructed EPR image of the DPPH phantom. Based on the linewidth, the spatial resolution of the EPRI of the DPPH was approximately $0.04 \mathrm{~cm}$. The phantom was composed of four diamond-shaped DPPH points, all of which can be distinguished in the image. Each point was positioned $2 \mathrm{~mm}$ (horizontally) and 4 mm (vertically) apart. The right-hand side of the phantom signal was very strong, as indicated by the areas in red 


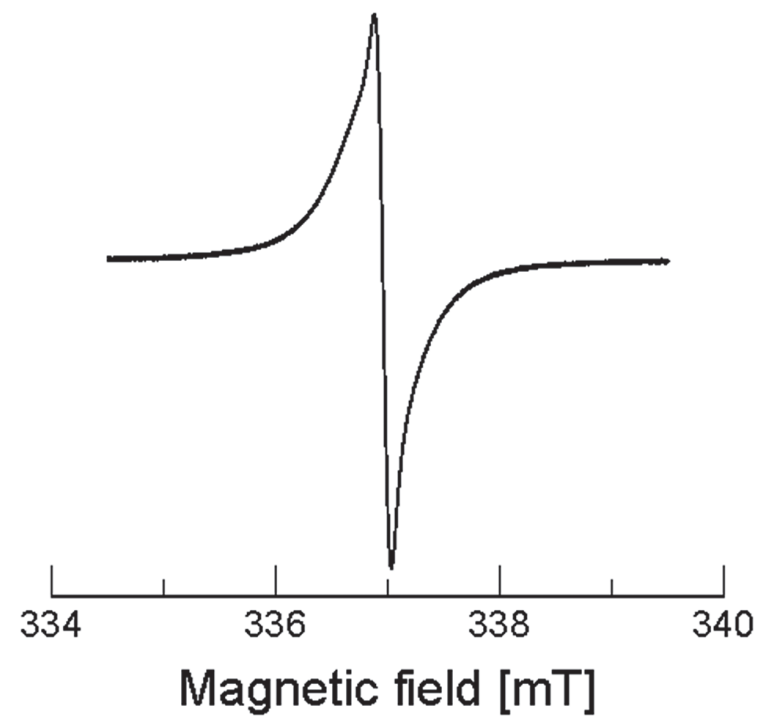

Fig. 2 EPR spectrum of the DPPH phantom obtained at a zero field gradient.

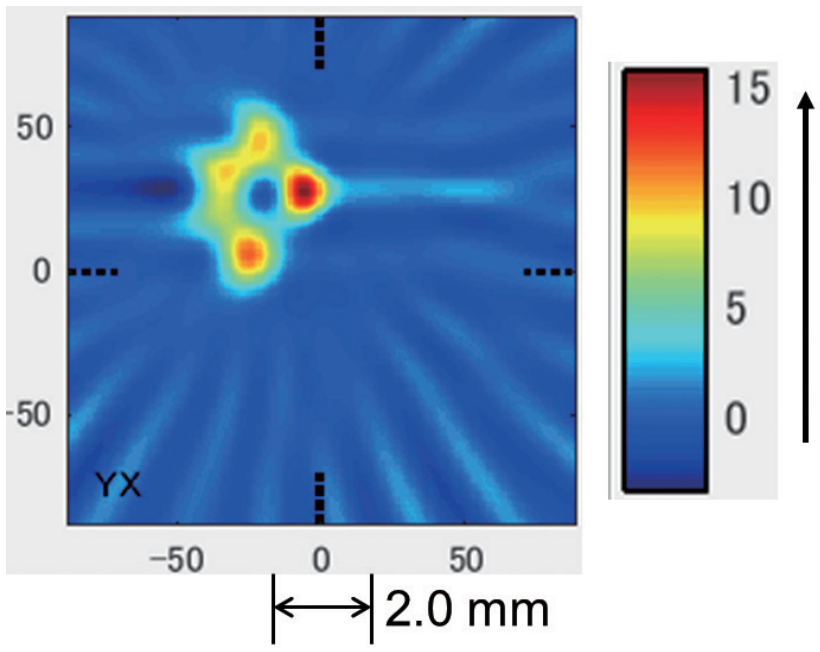

Fig. $32 \mathrm{D}$ image of the DPPH phantom consisting of four sample dots. The signal amplitude in arbitrary units is shown on the right.

color on the image, corresponding to the quantity of $\mathrm{DPPH}$ in each dot.

\subsection{CW EPR of the apple seeds}

Figure 4 shows the EPR spectrum of one entire apple seed. The EPR spectrum was composed of two distinguishable signals. These signals were stable, and the exact same results could be measured repeatedly for at least a month. One signal corresponded to $\mathrm{Mn}^{2+}$ species, whereas the other signal was assigned to an organic radical.

The first signal was characteristic of the $\mathrm{Mn}^{2+}$ paramagnetic center $\left(\mathrm{M}_{\mathrm{I}}=5 / 2\right.$-related sextet ${ }^{8)}, 100 \%$ natural abundance of ${ }^{55} \mathrm{Mn}$ isotope). The hyperfine coupling of the sextet was also consistent with the previously reported

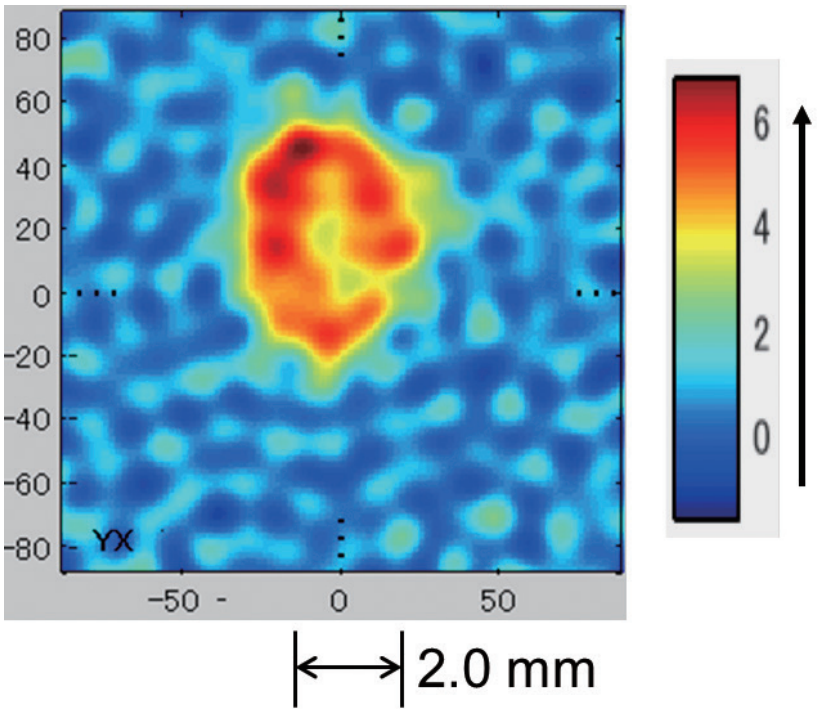

Fig. 6 2D EPR image of the whole seed. The signal amplitude in arbitrary units is shown on the right.

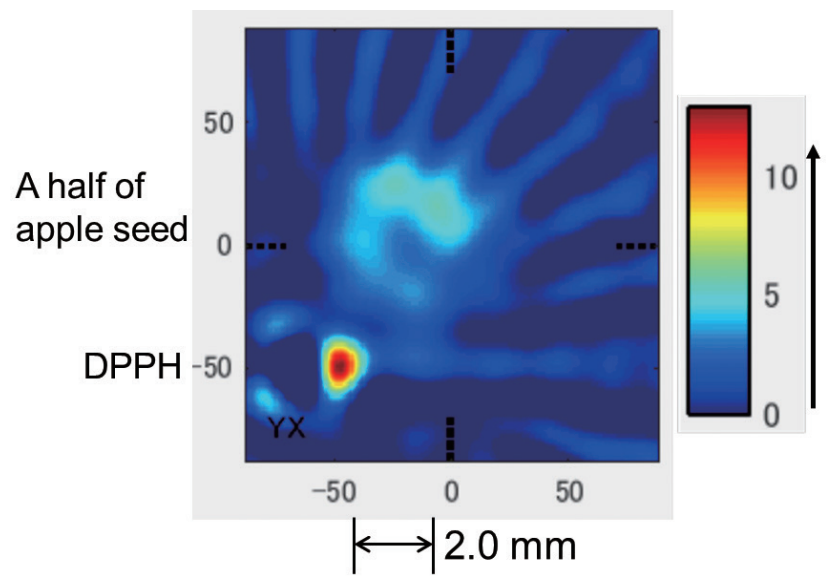

Fig. 8 2D EPR images of a half of the apple seed with DPPH as a reference signal.

value $^{8)}$. The apparent changes in hyperfine couplings from low to high fields, with coupling larger in high fields, were due to the overlap of multiple $\mathrm{Mn}^{2+}$ centers. A similar EPR spectrum was previously reported in black pepper seeds ${ }^{1}$.

The second signal was strong and reproducible. The relatively broad single-peak observed at $g \approx 2.00$ was indicative of stable organic carbon-centered radicals ${ }^{1,9)}$, indicating the possibility of the radical being generated under scavenging activities and that there are antioxidant-related organic compounds in the seed ${ }^{5}$. Thus, the observed signatures from these two different radical species were assigned as indicators of stable organic radicals and $\mathrm{Mn}^{2+}$ species.

\subsection{EPR imaging of apple seeds}

Figure 5 shows the EPR spectrum of one apple seed taken using the central region $(g \approx 2.00)$ of the EPR spec- 


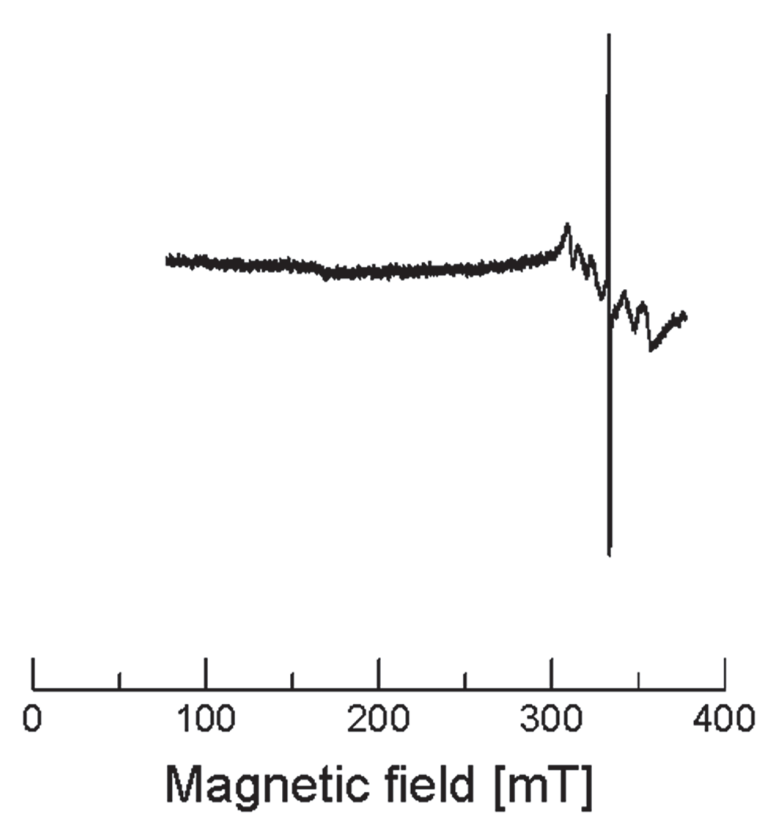

Fig. 4 EPR spectrum of a whole apple seed $(0.0316 \mathrm{~g})$, acquired at a $300 \mathrm{mT}$ magnetic field sweep width. The magnetic field center used was $227.0 \mathrm{mT}$.
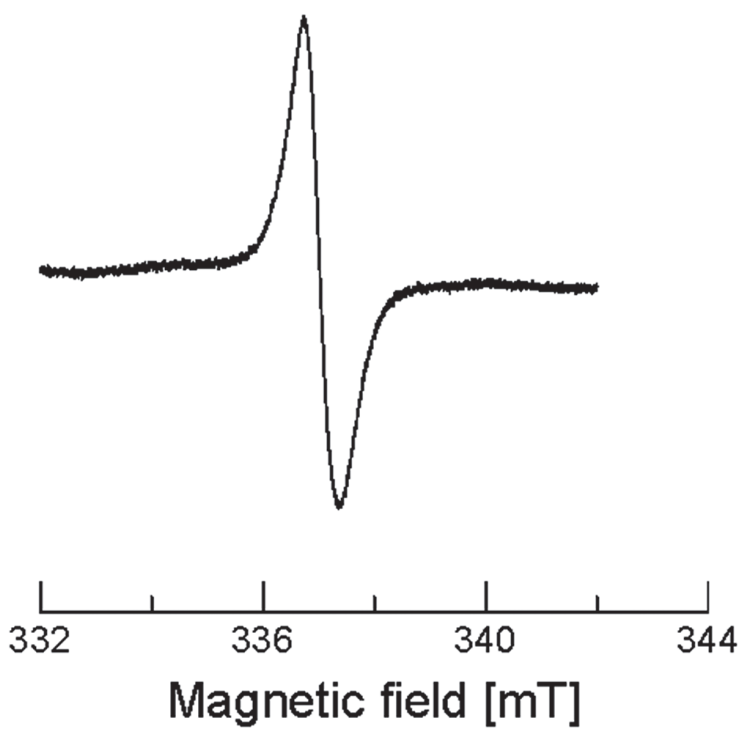

Fig. 5 EPR spectrum of a seed around $g \approx 2.00$, acquired with a $10.0 \mathrm{mT}$ sweep width. The spectrum was obtained with a single scan.

trum shown in Fig. 4. The peak-to-peak line width $\left(\Delta \mathrm{H}_{\mathrm{pp}}\right)$ of the signal was approximately $0.64 \mathrm{mT}$. Based on the line width, the spatial resolution of the EPRI of the seed was estimated to be $0.19 \mathrm{~cm}$. The concentration was estimated from the comparison to a TEMPOL (known concentration) solution in a capillary tube (outer diameter $1.0 \mathrm{~mm}$, inner diameter $0.9 \mathrm{~mm}$ ). The number of spins per gram was approximately $8 \times 10^{16}$.

To study the paramagnetic species present in apple

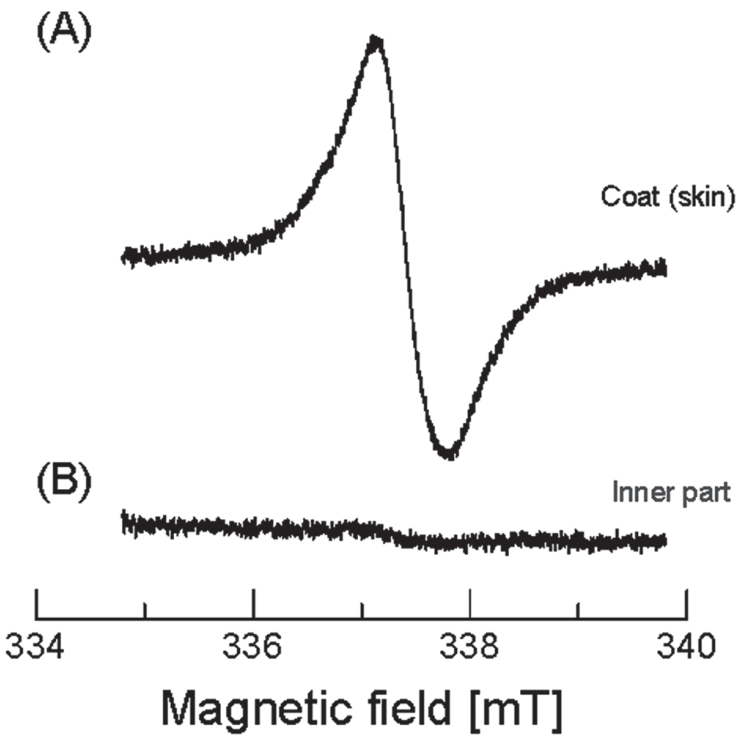

Fig. 7 CW EPR spectra of (A) the apple seed coat and (B) the inner part of the seed. Each spectrum was obtained in a single scan.

seeds, we performed EPRI of the seed. Figure 6 shows the EPR image from a whole seed, showing that the radical is located in the seed coat. The image was taken using a $5 \mathrm{mT}$ scan width. Very few radicals were observed in the cotyledon of the seed. Thus, the 2D EPR image suggests that the stable radical is located in the seed coat part of the seed.

To confirm the 2D EPRI observation, the seed coat and the inner part of the seed were measured separately. Figure 7 shows the CW EPR spectra of (A) the apple seed coat and (B) the inner part (cotyledon) of the seed. The EPR results from this model confirm that the stable radical is located in the seed coat. In addition, previous studies of methanol and hexane extracts of the seeds showed that the seeds contained various polyphenols and fatty acids ${ }^{5,6,10,11)}$. The stable radical in the seed can be produced during the scavenging activities of antioxidant compounds in the seeds. It is important to note that the seeds produce reactive oxygen species(ROS). ROS and reactive nitrogen species are involved in the regulation of various progresses in plants ${ }^{11}$. For example, ROS reacts with phenolic compounds $^{6)}$ and produces stable radical in the seed.

To examine the seed image in Fig. 6 in more detail, EPRI of a half of the apple seed was performed. The image produced, as shown in Fig. 8, clearly indicates the localization of stable radicals primarily in the seed coat. The signal in the lower bottom corner of the image corresponds to a DPPH standard, which was used to elucidate image intensity of the seed. The seed signal appears slightly weak due to the strong DPPH intensity. Here again, the EPR image confirms the relative intensity and location of radicals in the half seed.

It is important to note that the EPR images shown in 
Figs. 6 and 8 have slightly distorted-shapes. For example, the DPPH image in Fig. 8 has the slightly distorted-shape from a circular shape. One reason for this is the slight fluctuations in the static magnetic field stabilized by magnet current and not using a Hall probe. More specifically, for magnetic field monitoring, the NMR probe was removed to enable application of the magnetic field gradients for EPR images.

In summary, X-band EPR detected two different paramagnetic species in apple seeds. The distribution of the organic radical was imaged using noninvasive EPRI. EPRI showed that the stable radical is located in the seed coat, and not in the cotyledon of the seed. The present study showed that EPRI and EPR spectroscopy are very useful techniques for the evaluation of the distribution of stable paramagnetic species in biological samples.

\section{ACKNOWLEDGEMENTS}

Part of this research was supported by a Grant-in-Aid for Challenging Exploratory Research (JP15K12499) and for Scientific Research (B) (25282124) from the Japan Society for the Promotion of Science (JSPS) (K.N.). BE acknowledges NIH grants P41 EB002034, R50 CA211408 and partial support provided by the Program of Competitive Growth, Kazan Federal University.

\section{REFERENCES}

1) Nakagawa, K.; Epel, B. Location of radical species in a black pepper seed investigated by CW EPR and $9 \mathrm{GHz}$ EPR imaging. Spectrochim. Acta, Part A 131, 342346 (2014).
2) Nakagawa, K; Hara H. Investigation of radical locations in various sesame seeds by CW EPR and $9 \mathrm{GHz}$ EPR imaging. Free Radical Res. 49, 1-6 (2015).

3) Nakagawa, K; Ohba, Y; Epel, B; Hirata H. A 9 GHz EPR imager for thin materials: application to surface detection. J. Oleo Sci. 61, 451-456 (2013).

4) Epel, B.; Halpern, H.J. Electron paramagnetic resonance oxygen imaging in vivo. Electron Paramagnetic Resonance. Royal Soc. Chem. 23, 180-208(2013).

5) Lu, Y.; Foo, L.Y. Constitution of some chemical components of apple seed. Food Chem. 61, 29-33 (1998).

6) Walia, M.; Rawat, K.; Bhushan, S.; Padwada, Y.S.; Singh, B. Fatty acid composition, physicochemical properties, antioxidant and cytotoxic activity of apple seed oil obtained from apple pomace. J. Sci. Food Agric. 94, 929-934(2014).

7) The Center for EPR Imaging in vivo Physiology at the University of Chicago; http://epri.uchicago.edu.

8) Nakagawa, K. Effects of low dose X-ray irradiation of eggshells on radical production. Free Radical Res. 48, 679-683 (2014).

9) Weil, J.A.; Bolton, J.R.; Werltz, J.E. in Electron paramagnetic resonance, elementary theory and practical applications. John Wiley \& Sons, Inc. New York, p. 161, Chapter 7 (1994).

10) Xu, Y.; Fan, M.; Ran, J.; Zhang, T.; Sun, H.; Dong, M.; Zhang, Z.; Zheng, H. Variation in phenolic compounds and antioxidant activity in apple seeds of seven cultivars. Saudi J. Biol. Sci. 23, 379-388(2016).

11) Gniazdowska, A.; Krasuska, U.; Czajkowska, K.; Bogate, R. Nitric oxide, hydrogen cyanide and ethylene are required in the control of germination and undisturbed development of young apple seedlings. Plant Growth Regul. 61, 75-84(2010). 